\title{
Estimation of Brain Connectivity during Motor Imagery Tasks using Noise-Assisted Multivariate Empirical Mode Decomposition
}

\author{
Ki-Baek Lee*, Ko Keun Kim**, Jaeseung Song***, Jiwoo Ryu ${ }^{\S}$, \\ Youngjoo Kim ${ }^{\S}$ and Cheolsoo Park ${ }^{\dagger}$
}

\begin{abstract}
The neural dynamics underlying the causal network during motor planning or imagery in the human brain are not well understood. The lack of signal processing tools suitable for the analysis of nonlinear and nonstationary electroencephalographic (EEG) hinders such analyses. In this study, noiseassisted multivariate empirical mode decomposition (NA-MEMD) is used to estimate the causal inference in the frequency domain, i.e., partial directed coherence (PDC). Natural and intrinsic oscillations corresponding to the motor imagery tasks can be extracted due to the data-driven approach of NA-MEMD, which does not employ predefined basis functions. Simulations based on synthetic data with a time delay between two signals demonstrated that NA-MEMD was the optimal method for estimating the delay between two signals. Furthermore, classification analysis of the motor imagery responses of 29 subjects revealed that NA-MEMD is a prerequisite process for estimating the causal network across multichannel EEG data during mental tasks.
\end{abstract}

Keywords: Brain network, Partial directed coherence, Empirical mode decomposition, Electroencephalogram, Motor imagery

\section{Introduction}

Electroencephalogram (EEG), which is the most commonly used method for brain computer interface (BCI) studies, facilitates the implementation of computer-aided control by exclusively using brain activity due to its noninvasive nature and affordable recording equipment [1] [2][3]. In particular, the motor imagery (MI) paradigm, defined as the mental rehearsal of simple or complex motor acts that is not accompanied by overt body movements, has been widely used for EEG-based BCI systems [1]. MIbased BCI systems can be a novel interaction option for those with motor disabilities because they do not require voluntary muscle control [4]. The physiological basis for such an MI paradigm is the $\mathrm{mu}(8-12 \mathrm{~Hz})$ and beta rhythms $(18-25 \mathrm{~Hz})$ in the EEG, which are found in the motor cortex region of the brain when subjects imagine movement of their hands or fingers [5]. Currently, bandpass filters, such as infinite impulse response (IIR) filters, are often used to extract the power features in the frequency bands relevant to MI tasks [1]. However, this approach is still sub-optimal because it relies on Fourier analysis, which is

$\dagger$ Corresponding Author: Dept. of Computer Engineering, Kwangwoon University, Korea. (parkcheolsoo@kw.ac.kr)

* Dept. of Electrical Engineering, Kwangwoon University, Korea. (kblee@kw.ac.kr)

** Intelligence Lab., Convergence Center at LG Electronics, Korea. (kokeun.kim@gmail.com)

*** Dept. of Computer and Information Security, Sejong University, Korea. (jssong@sejong.ac.kr)

$\S$ Dept. of Computer Engineering, Kwangwoon University, Korea. (jwryu1128@gmail.com)

Received: May 27, 2015; Accepted: July 21, 2016 based on the assumption of linearity and the stationarity of the signal.

Recently, general brain function and communication between different regions of the brain were described based on an analysis of neural connectivity [4]. The functional connectivity that occurs during limb movement planning was also demonstrated by Li et al. [4] and Solodkin et al. [6]. The Granger causality, proposed for use in econometric models [7], is the most popular approach for detecting the directed influences between different activities [8]. Partial directed coherence (PDC) [9] is one of the best methods for determining such causal interdependencies, particularly in the frequency domain [8].

Many previous studies of power features and causal interdependency during MI tasks have employed conventional frequency decomposition techniques based on Fourier analysis, such as IIR filters [1]. Fourier analysis is based on predefined sets of basis functions, which results in poor time-frequency localization [10]. The linear basis functions of the Fourier analysis can be sub-optimal because the neurophysiological signals in the brain are nonlinear and nonstationary. In addition, EEG rhythms drift within different frequency bands, and thus, the Fourier method based on fixed bases is inadequate [11]. In this paper, we investigate the empirical mode decomposition (EMD) algorithm [10] to address the nonstationary and nonlinear neuronal signals, which is a fully data-driven time-frequency analysis algorithm. There is no prior assumption regarding the data in the process of EMD, making it suitable for the analysis of EEG [12].

Fourier analysis decomposes a multichannel EEG without 
considering cross-channel interdependence by applying fixed basis functions. The first version of EMD does not consider the cross-channel interdependence, which causes a uniqueness problem due to its data-driven approach, i.e., the components of each channel do not correspond in number and frequency [13]. Recently developed multivariate empirical mode decomposition (MEMD) approach can circumvent the uniqueness problem and produce more accurate time-frequency decomposition of the intrinsic oscillations in multichannel data than original EMD, when monocomponent oscillations of interest are shared by multiple channels [14].

Several studies have investigated the functional connectivity that occurs during MI tasks to demonstrate the ability of the connectivity feature to improve the performance of the MI BCI system [4, 5]. Furthermore, the EMD algorithm has been used to improve the accuracy of brain connectivity information during mental tasks $[8,15]$. However, most previous studies have used single-channel EMD algorithms to analyze multichannel EEG connectivity. This paper compares the performance of MEMD with the conventional bandpass filter and the other single-channel EMD algorithms to assess the connectivity feature of PDC. The simulation results of the synthetic and MI datasets suggest that MEMD is the optimal method for extracting accurate frequency components to estimate the functional connectivity of the brain.

\section{Methods}

\subsection{Empirical mode decomposition}

EMD is a suitable time-frequency decomposition algorithm for the analysis of nonlinear and nonstationary processes because it requires no prior assumptions regarding the data [10]. EMD decomposes a signal into amplitude modulation (AM) and frequency modulation (FM) components, called intrinsic mode functions (IMFs), using a data-driven technique. Two criteria of a signal that can be considered as an IMF are as follows: (1) the number of extrema and the number of zero crossings differ at most by one, and (2) the mean of the envelopes connecting the local maxima and local minima is approximately zero. The stopping criterion utilizes $a(t):=\left(e_{u}(t)-e_{l}(t)\right) / 2$ and the evaluation function $\sigma(t):=\left|\frac{m(t)}{a(t)}\right|$, and thus sifting is iterated until $\sigma(t)<\theta_{1}$ for some prescribed fraction (1$\alpha)$ of the total duration, while $\sigma(t)<\theta_{2}$ for the remaining fraction [19]. The procedures of EMD decomposition are outlined in Algorithm 1. After extracting the first IMF, $\mathrm{c}_{1}(\mathrm{t})$, this IMF is subtracted from the original signal, $v(t)$. The remnant, $r(t)$, becomes a new signal, and the same steps are repeated to $r(t)$ until no oscillations remain. Then, the original signal can be represented as
Algorithm 1. The standard EMD algorithm

1) Input signal, $v(t)$
2) Let $\tilde{v}(t)=v(t)$
3) Identify all local maxima and minima of $\tilde{v}(t)$
4) Find a lower 'envelope', $e_{l}(t)$ that interpolates all
local minima
5) Find an upper 'envelope', $e_{u}(t)$ that interpolates all
local maxima
6) Calculate the local mean, $\bar{m}(t)=\left(e_{l}(t)+e_{u}(t)\right) / 2$
7) Subtract the local mean from $\tilde{v}(t), c_{i}(t)=\tilde{v}(t)-$
$\bar{m}(t)(i$ is an order of IMF)
8) Let $\tilde{v}(t)=c_{i}(t)$ and go to step 3$)$; repeat until $c_{i}(t)$
becomes an IMF

$$
v(t)=\sum_{i=1}^{M} c_{i}(t)+r(t)
$$

where $c_{i}(t)$ are the IMFs and $r(t)$ is the residue.

Due to the nonlinear and nonstationary nature of EEG signal and noise, data-driven EMD has a critical problem for any analysis or data fusion of multichannel signals, namely the uniqueness problem. For instance, the different EEG channels could have different numbers and frequency components of IMFs, which hinders the analysis of the relationship across channels. In addition, similar frequencies occasionally appear across different IMFs, which is known as mode mixing. To solve these problems, $\mathrm{Wu}$ et al. proposed the ensemble EMD (EEMD) using a noiseassisted data analysis method [16]. EEMD defines the final IMF as the mean of an ensemble of IMFs produced by decomposing the signal added by white noise of finite amplitude. However, the uniqueness problem is not fully resolved by EEMD and is limited by its computational and univariate nature [5]. Rehman and Mandic [14] solved these problems in their development of MEMD, which is a natural extension of the original EMD. In the MEMD

\section{Algorithm 2. The multivariate EMD algorithm}

1) Choose a suitable point set for sampling on an (n-1) sphere

2) Calculate a projection, denoted by $\left\{p^{\theta_{k}}(t)\right\}_{t=1}^{T}$, of the input signal $\{\mathbf{v}(t)\}_{t=1}^{T}$ along the direction vector $\mathbf{x}^{\boldsymbol{\theta}_{\boldsymbol{k}}}$, for all $k$ (the entire set of direction vectors), giving $\left\{p^{\theta_{k}}(t)\right\}_{k=1}^{K}$ as the set of projections

3) Find the time instants $t_{j}^{\theta_{k}}$ corresponding to the maxima of the set of projected signals $\left\{p^{\theta_{k}}(t)\right\}_{k=1}^{K}$

4) Interpolate $\left[t_{j}^{\theta_{k}}, \mathbf{v}\left(\boldsymbol{t}_{\boldsymbol{j}}^{\boldsymbol{\theta}_{\boldsymbol{k}}}\right)\right]$ to obtain multivariate envelope curves $\left\{\mathbf{e}^{\theta_{k}}(t)\right\}_{k=1}^{K}$

5) For a set of $K$ direction vectors, the mean $\mathbf{m}(\mathrm{t})$ of the envelope curves is calculated as $\mathbf{m}(\mathrm{t})=\frac{1}{K} \sum_{k=1}^{K} \mathbf{e}^{\theta_{k}}(t)$

6) Extract the 'detail' $\mathbf{c}_{i}(t)=\mathbf{v}(\mathrm{t})-\mathbf{m}(\mathrm{t})$ ( $i$ is an order of IMF). If the 'detail' $\mathbf{c}_{i}(t)$ fulfills the stoppage criterion for a multivariate IMF, apply the above procedure to $\mathbf{v}(\mathrm{t})-\mathbf{c}_{\mathbf{i}}(t)$, otherwise apply it to $\mathbf{c}_{\mathbf{i}}(t)$ 
procedure, the local mean of $n$-dimensional signals are simultaneously estimated by projecting the signal along different directions in $n$-variate spaces [14]. Lowdiscrepancy Hammersley sequences are used to produce quasi-uniform points on high-dimensional spheres [17], by which a uniform set of direction vectors to project the signal can be obtained. Algorithm 2 outlines the steps of MEMD. ${ }^{1}$ When all of the $\mathrm{K}$ projected signals fulfill the stopping criteria ${ }^{2}$ used in the original EMD, the sifting process of MEMD is stopped [14].

Rehman and Mandic [18] introduced a noise-assisted MEMD (NA-MEMD), which exploits the properties of MEMD; a dyadic filter bank on each channel when multidimensional white Gaussian noises (WGNs) are decomposed using MEMD. Using the composite signal of multivariate data and multivariate independent white noise, the noise is never mixed with the data channels when creating a filter bank structure for the data. This approach provides a more accurate definition of the frequency bands inherent in the data [11]. Due to the noise channel, the frequency components in IMFs obey the dyadic filter bank property, which ensures that IMFs corresponding to the original input data are aligned and yield the same frequency information at each level of IMFs. In this paper, we use NA-MEMD for MEMD decomposition because it was shown to outperform the MEMD in [5].

\subsection{Partial directed coherence}

C.W.J. Granger [7] developed Granger causality to measure the causal relationship between two signals. Given past observations of signal $\mathrm{X}$ with past observations of signal $\mathrm{Y}$, the causal inference from $\mathrm{X}$ to $\mathrm{Y}$ can be determined by reducing the prediction error of signal $\mathrm{Y}$. Conditional Granger causality was developed to determine whether the relationship between two data points is direct or mediated by a recorded time series [4].

Partial directed coherence (PDC) [21] has been developed as an extension of conditional Granger causality in the frequency domain. Using a multivariate autoregressive (MVAR) model, the multi-channel data, $\mathrm{X}(\mathrm{t})$, can be described as follows:

$$
X(\mathrm{t})=\sum_{\tau=1}^{p} A(\tau) X(t-\tau)+N(t)
$$

where $N(\mathrm{t})$ denotes the prediction error of the model. Then, this equation can be rearranged as an input-output relationship as follows:

$$
N(\mathrm{t})=\sum_{\tau=0}^{p} \hat{A}(\tau) X(t-\tau)
$$

where

\footnotetext{
${ }^{1}$ The MATLAB code of MEMD is available from 'www.commsp. ee.ic.ac.uk/\%mandic/research/emd.htm'.

${ }^{2}$ We employed a combination of EMD stoppage criteria, given in [19] and [20], for MEMD sifting.
}

$$
\hat{A}(\tau)=\left[\begin{array}{c}
I, \tau=0 \\
-A(\tau), \tau>0
\end{array}\right]
$$

(3) can be presented in the frequency domain as follows:

$$
\begin{aligned}
& N(f)=A(f) X(f) \\
& A(f)=\sum_{\tau=0}^{p} \hat{A}(\tau) e^{-j 2 \pi f \tau}
\end{aligned}
$$

where $f$ is the frequency. Based on $A(f)$, the PDC can be calculated as

$$
\pi_{i j}(f)=\frac{a_{i j}(f)}{\sqrt{\sum_{k=1}^{C}\left|a_{k j}(f)^{2}\right|^{2}}}(7)
$$

$\pi_{\ddot{j}}(f)$ denotes the estimation of causal inference from $X_{j}$ to $X_{i}$ at frequency $f$. This measurement can be interpreted as the normalized causal outflow from $X_{j}$ to $X_{i}$ by the total amount of those from $X_{j}$ [4], and thus,

$$
\sum_{i=1}^{C} \pi_{\ddot{j}}(f)=1
$$

where $C$ represents the number of channels except channel $j$. Therefore, a higher $\pi_{i j}$ indicates that signal $X_{i}$ is more affected by signal $X_{j}$ among all of the signals influenced by signal $X_{j}$.

\section{Numerical Simulation}

To verify the performance of the NA-MEMD compared with the other EMD methods (EEMD and EMD) and conventional bandpass filtering method like IIR filter and continuous wavelet transform (CWT), we tested two synthetic dataset with time-delayed coupling. The single channel algorithms, EEMD and EMD, were used to test the advantage of the multichannel algorithm, NA-MEMD. In addition, CWT was designed for the analysis of nonstationary data [10] and thus its performance was compared with that of NA-MEMD.

\subsection{Design of synthetic dataset}

Firstly, we used two autoregressive processes of order 2 (AR(2)), which had a unidirectional time-delayed coupling. The synthetic data were defined as below

$$
\begin{gathered}
\mathrm{x}_{1}(t)=0.95 \sqrt{2} x_{1}(t-1)-0.9025 x_{1}(t-2) \\
+v_{1}(t), \\
\mathrm{x}_{2}(t)=0.64 \sqrt{2} x_{2}(t-1)-0.6025 x_{2}(t-2) \\
-0.07 x_{1}(t-40)+v_{2}(t)
\end{gathered}
$$

where the time delay of $x_{1}(t)$ affecting $x_{2}(t)$ is 40 , and 
$v_{1}(t)$ and $v_{2}(t)$ are different realisations of white Gaussian random noises with signal-to-noise ratio (SNR) 0 $\mathrm{dB}$. The sampling frequency was $80 \mathrm{~Hz}$ and the data length 1000. The power spectra of $x_{1}(t)$ and $x_{2}(t)$ were calculated using periodogram with Bartlett window, and they are illustrated in Fig. 1 (a) and (b). As can be seen, they have broad peaks centered at $10 \mathrm{~Hz}$. Owing to these spectral properties, strong PDC for the time-delayed coupling data can be expected between $1 \mathrm{~Hz}$ and $30 \mathrm{~Hz}$. In order to investigate the directionality of the interaction between two data, the data points of the time series were shifted by one point, and the maxima PDC, $\pi_{21}^{m a x}$, on the time delay $\tau$ are calculated for every shift. Due to the time delay 40 in Eq. (9), the highest PDC can be expected around $\tau=40$.

Secondly, three autoregressive processes of order 2 $(\mathrm{AR}(2))$ were used, which had both unidirectional and bidirectional time-delayed couplings. The synthetic data were defined as below,

$$
\begin{gathered}
x_{1}(t)=0.64 \sqrt{2} x_{1}(t-1)-0.6025 x_{1}(t-2) \\
-0.07 x_{3}(t-60)+v_{1}(t), \\
x_{2}(t)=0.64 \sqrt{2} x_{2}(t-1)-0.6025 x_{2}(t-2) \\
-0.07 x_{1}(t-60)+v_{2}(t), \\
x_{3}(t)=0.64 \sqrt{2} x_{3}(t-1)-0.6025 x_{3}(t-2) \\
-0.07 x_{1}(t-60)+v_{3}(t),
\end{gathered}
$$

where the time delay of $x_{1}(t)$ affecting $x_{2}(t)$ and $x_{3}(t)$ is 60, and that of $x_{3}(t)$ affecting $x_{1}(t)$ is 60. $v_{1}(t)$, $v_{2}(t)$ and $v_{3}(t)$ are different realisations of Gaussian random noises with signal-to-noise ratio (SNR) $0 \mathrm{~dB}$. The sampling frequency and data length are the same as two AR process model. $x_{1}(t)$ affects $x_{2}(t)$ unidirectionally, and $x_{1}(t)$ and $x_{2}(t)$ affect each other bidirectionally corresponding to the models. Based on the periodograms of these signals, the frequency band between $1 \mathrm{~Hz}$ and $30 \mathrm{~Hz}$ is the range of interest expecting strong PDC. In addition, the highest PDC can be expected around $\tau=60$ corresponding to the design of the AR processes.

\subsection{Estimation of connectivity}

Based on the power spectral density (PSD) of the synthetic dataset in Fig. 1 (a) and (b), we extracted the frequency components between $1 \mathrm{~Hz}$ and $30 \mathrm{~Hz}$ for both two and three AR processes using conventional IIR filter ( $5^{\text {th }}$-order Butterworth), $\mathrm{CWT}^{3}$ (widely used Morlet wavelet [10]), original EMD, EEMD and NA-MEMD. The standard single channel EMD algorithms (EMD, EEMD) were applied to each channel separately, while NA-MEMD was applied to the two/three channel data and one

${ }^{3} \mathrm{CWT}$ is given by $\overline{\mathrm{W}}(\breve{\mathrm{a}}, \breve{\mathrm{b}} ; \mathrm{V}, \varphi)=|\check{\mathrm{a}}|^{-1 / 2} \int_{-\infty}^{\infty} \mathrm{V}(\mathrm{t}) \varphi^{*}\left(\frac{\mathrm{t}-\breve{b}}{\breve{a}}\right) \mathrm{dt}$, where $\varphi^{*}$ is the mother wavelet, ă the dilation factor and $\breve{b}$ the translation of the origin.

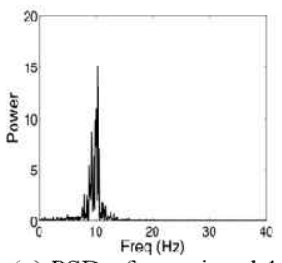

(a) PSD of raw signal 1

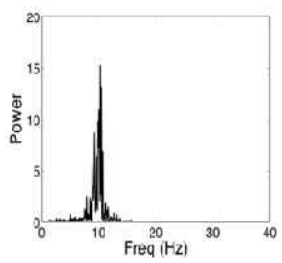

(c) PSD using IIR for signal 1

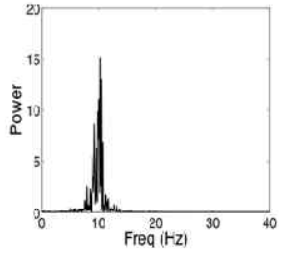

(e) PSD using EMD for signal 1

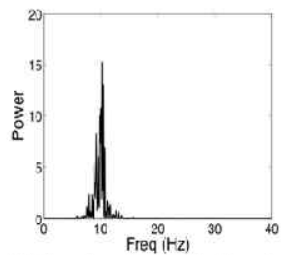

(g) PSD using EEMD for signal 1

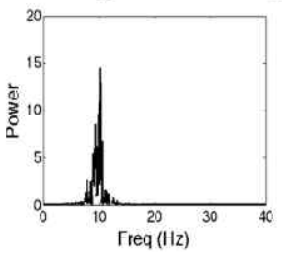

(i) PSD using NA-MEMD for signal 1

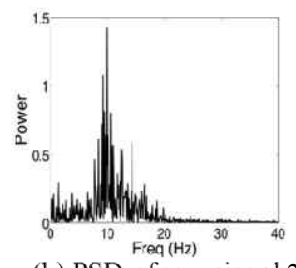

(b) PSD of raw signal 2

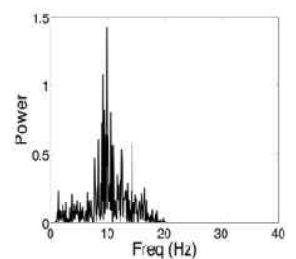

(d) PSD using IIR for signal 2

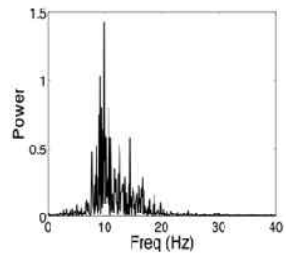

(f) PSD using EMD for signal 2

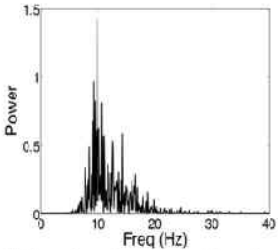

(h) PSD using EEMD for signal 2

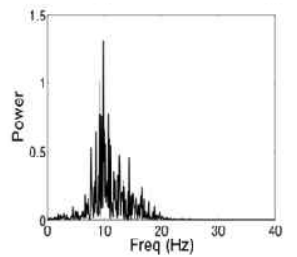

(j) PSD using NA-MEMD for signal 2
Fig. 1. Power spectral density of raw signals produced using (9) and (10) and their bandpass-filtered signals. (a) and (b) show the PSD of the raw signals, and (c)-(j) show the bandpass-filtered signals using the IIR filter, EMD, EEMD and NA-MEMD

additional noise channel (SNR $20 \mathrm{~dB}$ ) simultaneously. An investigation of the spectral information based on the periodogram indicated that EMD and EEMD have frequency components in their first and second IMFs, and NA-MEMD has frequency components in its second and third IMFs. The power spectra of the extracted components using the four methods for two AR processes are displayed in Fig. 1 (c)-(j). Using the extracted frequency components, PDCs were calculated with shifting the data points of the time series by one point. Due to the design of the dataset, the peak of the PDC around $\tau=40$ for two AR processes and $\tau=60$ for three AR processes can be expected. The peakedness would tell the performance of the frequency 


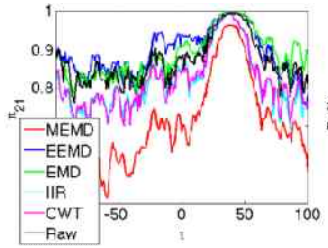

(a) $\operatorname{PDC} \pi_{21}^{\max }(\tau)$

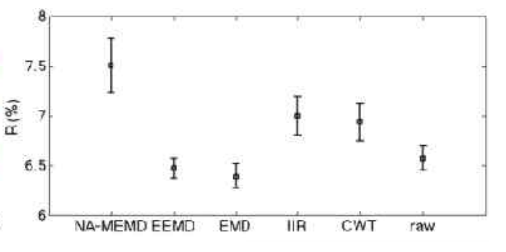

(b) $\mathrm{R}$ for $\pi_{21}^{\max }(\tau)$
Fig. 2. (a) The dependencies of the maximal values of the PDC on the time delay between two synthetic data produced using Eq. (9) and (10) and different filtering approaches. The time delay used in the simulations is $\tau=40$ and 20 synthetic data with different realisation of white Gaussian noise were used to produce the averaged results. Sharper peak around 40 in the plot represents better performance of the method. Note NA-MEMD outperforms the other methods. (b) Performance index, $\mathrm{R}$ in Eq. (14), of the different filtering methods for the time delay simulation in (a). The error bars illustrate standard errors of 20 trials with different realisations of white Gaussian noise. Note NAMEMD outperforms the other method, whose performance is significantly higher than the others ( $<<0.01$ using the one-tailed t-test and ANOVA 1way analysis)

decomposition algorithms, that is, the sharper the peak is, the better the algorithm is. Specifically, we calculated the ratio between the sum from $\tau=0$ to $\tau=100$ and those from $\tau=37.5$ to $\tau=42.5$ ( $5 \%$ of the range) for two AR processes (see Eq. (13)) and from $\tau=57.5$ to $\tau=62.5$ for three AR processes,

$$
\mathrm{R}=100 \times \frac{\sum_{\tau=37.5}^{42.5} \pi_{21}^{m a x}(\tau)}{\sum_{\tau=0}^{100} \pi_{21}^{m a x}(\tau)}
$$

The performance index for an accurate estimation of PDC will therefore give high percentage while worse results will approach zero. The PDC graphs obtained using the raw data and frequency components using the four algorithms were calculated and averaged over 20 independent trials with different realisations of white Gaussian noise.

Fig. 2 (a) illustrates the averages of $20 \pi_{21}^{\max }(\tau)$ graphs for two AR processes and shows the sharper peak of NAMEMD compared to the others including that of raw signal. The standard errors (error bars) of the performance indices, $\mathrm{R}$, using all methods including raw signal are shown in Fig. 2 (b). Overall, the average performance index using NAMEMD were the highest. For rigour, the higher performance index using NA-MEMD compared to the others was analysed using the one-tailed t-test for pairwise testing and ANOVA 1-way analysis for the six group testing, and the significance of the higher performance using NA-MEMD was verified with p-values less than 0.01 by both statistical tests.

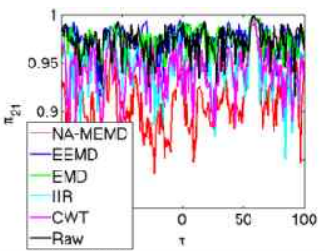

(a) $\operatorname{PDC} \pi_{21}^{\max }(\tau)$

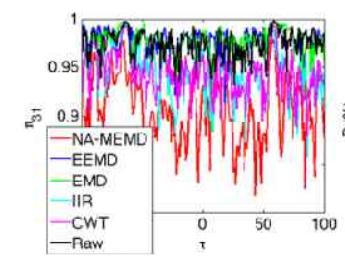

(c) $\operatorname{PDC} \pi_{31}^{\max }(\tau)$

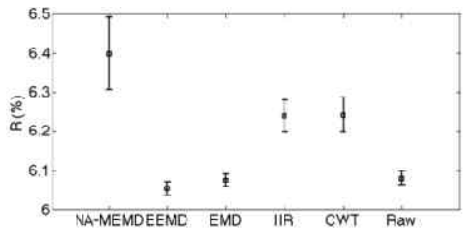

(b) $\mathrm{R}$ for $\pi_{21}^{\max }(\tau)$

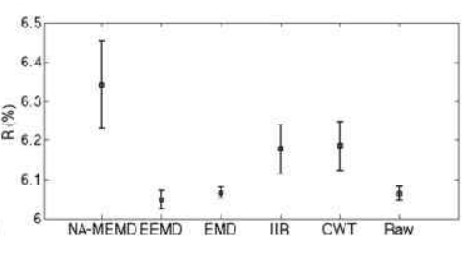

(d) $\mathrm{R}$ for $\pi_{31}^{\max }(\tau)$
Fig. 3. (a), (c) The dependencies of the maximal values of the PDC on the time delay between two synthetic data for unidirectional (a) and bidirectional models (c), produced using Eq. (11)-(13) and different filtering approaches. Note NA-MEMD outperforms the other methods with sharper peak around 60. (b), (d) Performance index, R, of the different filtering methods for the time delay simulations in (a) and (c). The error bars illustrate standard errors of 20 trials with different realisations of white Gaussian noise. Note NA-MEMD outperforms the other method, whose performance is significantly higher than the others $(\mathrm{p}<0.01$ using the one-tailed t-test and ANOVA 1-way analysis) for both unidirectional (b) and bidirectional models (d)

Fig. 3 displays the PDC results of three AR processes between Eq. (11) and Eq. (12), unidirectional relation, and between Eq. (11) and Eq. (13), bidirectional relation. The results are the averages of $100 \pi_{21}^{\max }(\tau)$ and $\pi_{31}^{\max }(\tau)$ with different realisation of white Gaussian random noise. Due to the directionality, $\pi_{21}^{m a x}(\tau)$ has a peak around $\tau=$ 60 while $\pi_{31}^{m a x}(\tau)$ has two around $\tau=60$ and $\tau=-60$. In this simulation, NA-MEMD consistently produces the best performance with the sharpest peak, which is validated by the performance index, R, in Fig. 3 (b) and (d). One-tailed t-test and ANOVA 1-way analysis verify the significant improvement of NA-MEMD to estimate the PDC compared to the other methods with $\mathrm{p}$-values less than 0.01 by both tests.

In order to investigate the performance of NA-MEMD depending on the noise condition in additional channels, different SNR of noise and number of noise channels were tested for the same simulation as Fig. 2. As can be seen in Fig. 4, NA-MEMD consistently outperforms the other algorithms in all noise conditions with p-values less than 0.01 by ANOVA 1-way analysis.

\section{Application to a MI EEG Dataset}

In this section, a real-world MI EEG dataset has been 


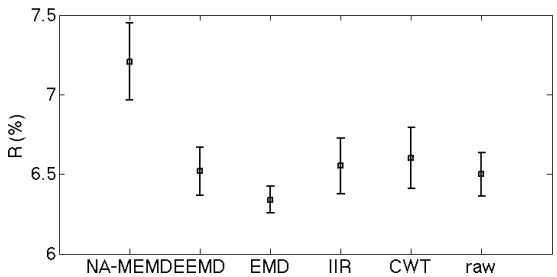

(a) $10 \mathrm{~dB}$

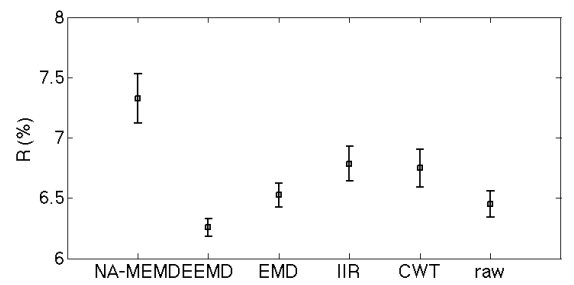

(c) 2 Noise

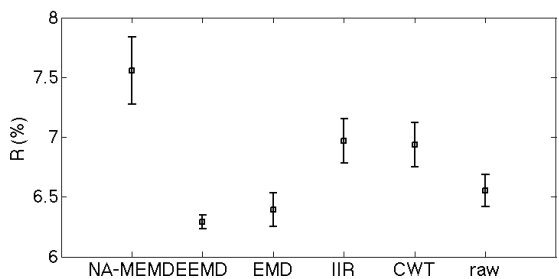

(b) $30 \mathrm{~dB}$

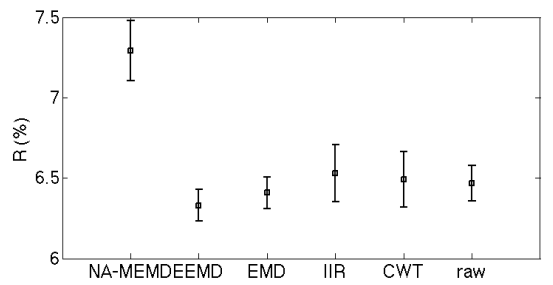

(d) 3 Noise

Fig. 4. Studies of NA-MEMD performance depending on the amount of noise in the additional channel and the number of noise channels. (a) (b) NA-MEMD used $10 \mathrm{~dB}$ and $30 \mathrm{~dB}$ noise in the additional noise channel for the same simulation as Fig. 2. (c) (d) NA-MEMD used two and three additional noise channels. Note that NA-MEMD outperforms the others in all the scenarios regardless of the noise conditions

used to demonstrate that NA-MEMD outperforms the other EMD algorithms, the conventional bandpass IIR filter and CWT.

\subsection{EEG dataset}

To investigate the performance of NA-MEMD for PDC estimation, we used the Physiobank Motor/Mental Imagery (MMI) database recorded using the BCI2000 system [22], which is available through Physionet ${ }^{4}$ [23]. The datasets contain 64-channel EEG recording of 100 subjects according to the 10-10 system, sampled at $160 \mathrm{~Hz}$, while they performed $45 \mathrm{MI}$ tasks. The data blocks for left hand and right hand movement imagination for a duration of $4 \mathrm{~s}$ were chosen for the PDC analysis. Although multiple regions of the scalp were monitored to improve the performance of BCI systems, a large number of EEG electrodes may cause ambient noise and redundant signals resulting in poor performance of BCI system. Additionally, multichannel EEG configuration delays the use of the wearable $\mathrm{BCI}$ devices due to the inconvenience of donning it. In terms of the real-time BCI, selecting the least number of channels is important to reduce the computational complexity [24]. Of the $64 \mathrm{EEG}$ channels, the 12 shown in Fig. 11-13 were selected for the proposed analysis, Fp1, Fp2, F3, F4, T7, T8, C3, C4, P3, P4, O1 and O2 representing all the functional regions of the scalp, frontal polar, frontal, temporal, central, parietal and occipital. In addition, the study of the functional network during MI demonstrated brain networks underlying the mental behavior on the M1 (primary motor cortex), S1 (primary and secondary somatosensory cortices), LPMC (lateral

\footnotetext{
${ }^{4} \mathrm{http} / / /$ www.physionet.org/pn4/eegmmidb/\#experimental-protocol
}

premotor cortex, dorsal), SMA (supplementary and presupplementary motor areas), CRB (cerebellum), PAR (superior parietal lobule and intraparietal sulcal area), IF (inferior frontal cortex, LPMC ventral; inferior frontal gyrus and anterior insular cortex) and OCC (occipital lobe) using correlation analysis [6], which were covered with the chosen 12 electrodes.

\subsection{Preprocessing}

The EEG signals during the MI tasks were bandpass filtered to occupy the $8-30 \mathrm{~Hz}$ frequency band [25][26] using the IIR filter and subsequently CWT, EMD, EEMD and NA-MEMD. EMD and EEMD were applied to each channel separately, whereas NA-MEMD was applied to the 12-channel data and two additional noise channel (SNR of $20 \mathrm{~dB}$ ) simultaneously. The IMFs relevant to the MI tasks were retained or omitted based on inspection; that is, the most relevant IMFs were selected to obtain the filtered signals. The relevant IMFs were estimated based on the best separation performance with respect to the classification algorithm for two tasks. The second IMF obtained using EMD has the frequency components related to the MI tasks, whereas the second and third IMFs of EEMD have them. NA-MEMD produced the frequency components related to the MI tasks in its third and fourth IMFs.

\subsection{PDC feature extraction}

The PDC features relevant to MI responses were extracted using principal component analysis (PCA) to reduce the dimensionality. PCA has been used to extract features with cancelling noise or irrelevant information to 
the purpose of the system in various areas. Lugger et al. used PCA to extract 6 features from 16 adaptive autoregressive parameters of EEG signals for the discrimination of MI tasks [27], and Noori et al. applied PCA for the 5 input features to support vector machine (SVM) classifier from 18 variables of weather for prediction of stream flow [28]. There are 66 PDC features for the 12 channels due to the combination of the paired channels among them, which would cause the negative effect of dimensionality [29]. Therefore, the dimensionality of the $1 \times 66$ vector $\left[\pi_{1,2}^{\max }, \pi_{1,3}^{\max }, \ldots, \pi_{11,12}^{m a x}\right]$ was reduced to a $1 \times 2$ feature vector, $\mathbf{f}=\left[f_{1}, f_{2}\right]$, using PCA. The PDC feature vectors were calculated for all five bandpassfiltered datasets using the IIR filter, CWT, EMD, EEMD and NA-MEMD

\subsection{Classification}

Five bandpass-filtered datasets using the IIR filter, CWT, EMD, EEMD and NA-MEMD were classified into the two MI task groups using a SVM [30], and their performances were compared. An algorithm obtaining higher classification accuracy produced more accurate PDC estimations corresponding to the left and right MI tasks. A SVM with a Gaussian kernel ${ }^{5}$ was used to classify the PDC feature vector $\mathbf{f}=\left[f_{1}, f_{2}\right]$. The datasets were divided into training and testing sets, with the former containing $80 \%$ of the data and the latter containing $20 \%$ of the data. The classification procedure was repeated 5 times while changing the sample order (5-fold cross-validation), and the average of these outcomes was the final classification rate. The process of our experiments is illustrated in Fig. 5 using a block diagram.

\subsection{Results}

The classification accuracies estimated using NAMEMD, EEMD, EMD, the IIR filter and CWT were compared and are displayed in Fig. 6. The confidence interval between two classes has an upper limit of $64.0 \%$

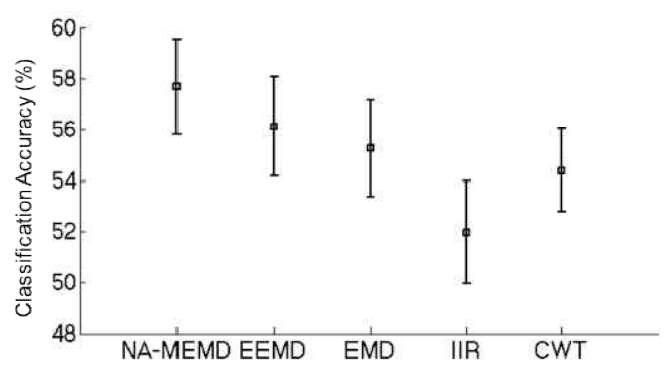

Fig. 5. Block diagram of the benchmark test of 6 different frequency filtering algorithms for the estimation of PDC

\footnotetext{
${ }^{5}$ The MATLAB code can be downloaded from [31]
}

for 45 trials $^{6}$. Among the 100 subjects in the Physiobank MMI database, the subjects for whom the classification results were below 64\% using NA-MEMD, EEMD, EMD, the IIR filter and CWT were rejected. The variation of the performance in the MI tasks has been reported, claiming some target users could not produce distinguishable brain signal during the tasks [33]. In particular, subjects performed poorly had less developed brain network [33]. Therefore, low-performance subjects were discarded for the fair benchmark test of the PDC estimation methods. Based on the confidence interval criterion, 29 subjects were chosen as significant subjects, and Fig. 6 shows the average classification rates across the subjects with standard errors (error bars). The average classification rates using NA-MEMD were the highest overall. Fig. 7 (a)(d) display a series of scatterplots of the classification results between the conventional IIR filter and one of the other algorithms using the results in Fig. 6. If the performances of the two algorithms are the same, the dots in the plot lie on the diagonal. However, many of the dots in Fig. 7 (a) lie above the diagonal, indicating that NA-MEMD outperformed the conventional IIR filter, whereas EEMD, EMD and CWT did not outperform the conventional IIR filter, as shown in Fig. 7 (b), (c) and (d). For rigor, the difference in the classification rates between the two algorithms was analyzed using a one-tailed t-test. The corresponding p-value in Fig. 7 (a) demonstrates that NA-MEMD performs significantly better than the IIR filter (p-value less than 0.05). In contrast, EEMD, EMD and CWT do not have significant p-values, as the data points in the scatterplots are distributed around the diagonal.

In order to investigate the performance of PCA for the feature extraction of $\mathrm{PDC}$, the proposed process without the PCA has been conducted. Fig. 8 displays the classification results using the five filtering algorithms with

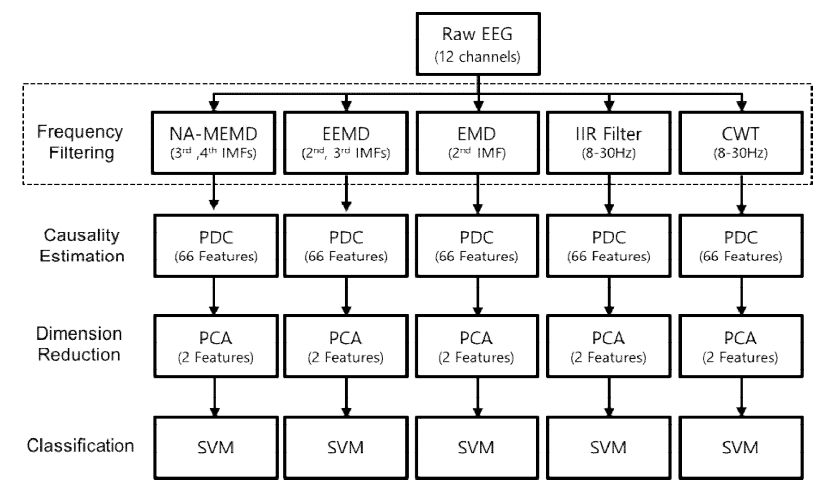

Fig. 6. Classification results of MI tasks for 29 subjects using the five different filtering methods. The error bars indicate the standard errors for all subjects. Only NA-MEMD outperforms the conventional IIR filter significantly $(\mathrm{p}<0.05)$

\footnotetext{
${ }^{6}$ The chance level in a two class separation is not $50 \%$ but $50 \%$ with a confidence interval depending on the trial number [32].
} 
PCA (blue bars) and without PCA (red bars). Note the big difference between those results, indicating the effect of PCA to extract the features among all the PDC values. In particular, NA-MEMD and CWT had the significant improvement using PCA $(\mathrm{p}<0.05)$.

Next, we developed a histogram of the subjects whose best classification accuracies were obtained using one of the EMD algorithms, the conventional IIR filter or CWT in Fig. 9. As seen in Fig. 9 (a), the IIR filter and CWT yielded the best performance for only 5 subjects each, whereas one of the EMD algorithms yielded the best performance for the other 19 subjects. In particular, the pairwise comparisons between the IIR filter and one of the other algorithms are

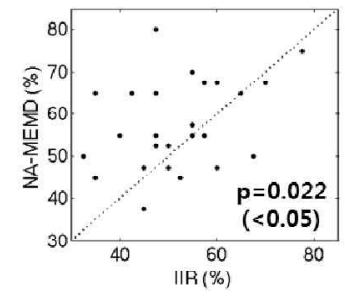

(a) NA-MEMD vS IIR

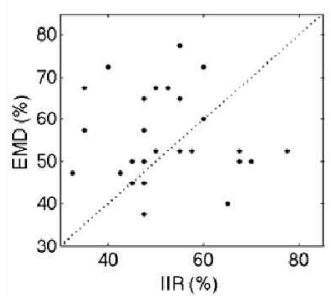

(c) HMI) vs IIR

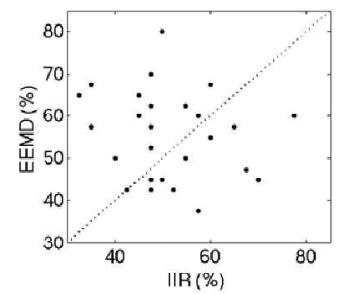

(b) EEMD vs IIR

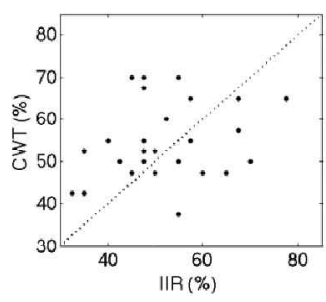

(d) CWI vs IIR
Fig. 7. Scatterplot of the classification results obtained using the PDC features of NA-MEMD, EEMD, EMD, the IIR filter and the CWT. Data points above the diagonal indicate the cases in which the other methods outperform the IIR filter. The superior performance of NA-MEMD was verified by a onetailed t-test $(p<0.05)$. (a) NA-MEMD versus the IIR filter, (b) EEMD versus the IIR filter, (c) EMD versus the IIR filter, (d) CWT versus the IIR filter

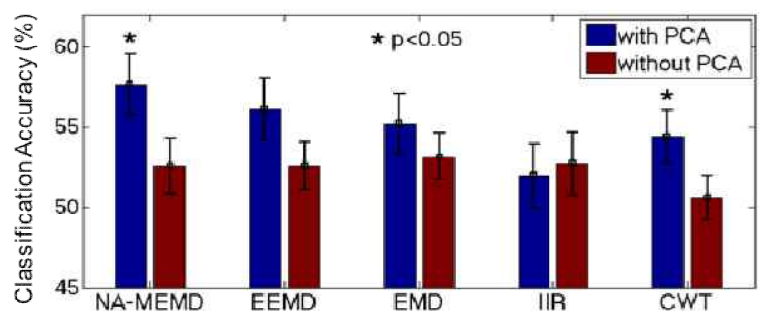

Fig. 8. Classification results of MI tasks for 29 subjects using the five different filtering methods with PCA (blue bars) and without PCA (red bars). The error bars indicate the standard errors for all subjects, and '*' was presented when the method with PCA outperformed that without PCA significantly $(\mathrm{p}<0.05)$. shown in Fig. 9 (b), where the number denotes the number of subjects who obtain the better results using one of the EMD algorithms and CWT than the IIR filter. NA-MEMD yields the best performance with the largest number of subjects among all three EMD algorithms and CWT, demonstrating that it is the best algorithm to estimate PDC features.

A receiver operating characteristic (ROC) analysis [34] was also used to assess the classification performance of the PDC features using the different frequency decomposition algorithms, plotting the sensitivity (true positive rate) of the classification against the false-positive rate. For instance, when we discriminate between left hand and right hand MI tasks, the sensitivity refers to the percentage of trials that were classified as a left hand MI task among all left hand MI tasks and the false-positive rate refers to the percentage of trials that were classified as a left hand MI task among all right hand MI tasks. The area under the ROC curve (AUROC) measures how well the PDC features of two MI tasks can be distinguished. A large AUROC indicates that the sensitivity increases rapidly with an increasing threshold for the classifier but increases more gradually for the false positive results. In contrast, a small AUROC indicates that the sensitivity increases

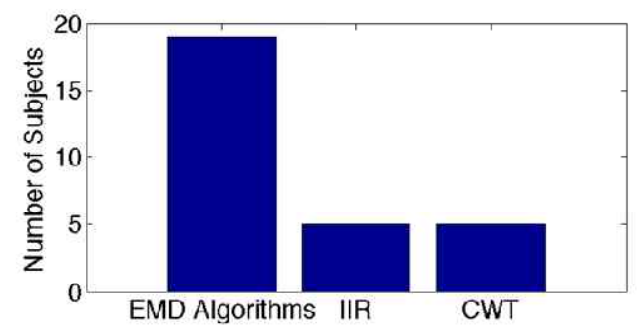

(a) EMD algorithms vs IIR

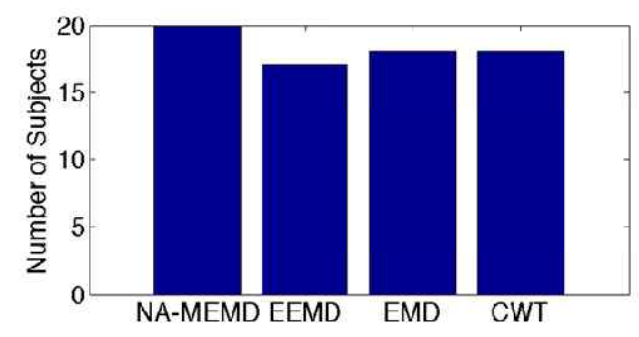

(b) Comparison of the EMD algorithms and CWT

Fig. 9. The number of subjects whose best classification rates were obtained using one of the EMD algorithms, the IIR filter and the CWT. (a) The EMD algorithms largely outperform the conventional IIR filter, suggesting that EMD methods could be more suitable for estimating PDC features than the conventional method. (b) Pairwise comparison between the IIR filter and one of the EMD algorithms and the CWT, illustrating that NAMEMD is the most suitable method, as more subjects have better performance using NA-MEMD rather than the other single channel algorithms 
gradually as the classifier threshold increases with a steep increase in false- positive results. The difference between the AUROCs of NA-MEMD, EEMD, EMD, the IIR filter and CWT were investigated, and Fig. 10 (a) displays the average ROC curves for the classification performance of all 29 significant subjects. The AUROC for NA-MEMD is larger than the AUROCs for the other approaches. To compare the AUROCs of all subjects for the different methods, the distributions of the results are shown in Fig. 10 (b) with standard errors (error bars). This analysis confirms that NA-MEMD outperforms the other methods.

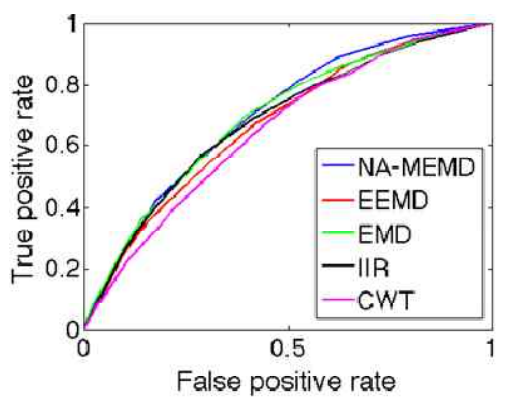

(a) ROC curves

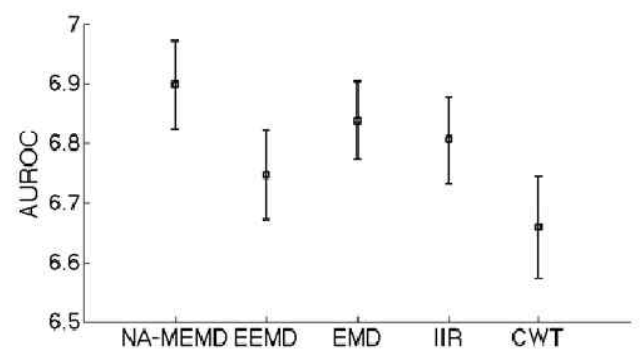

(b) Standard errors of AUROCs

Fig. 10. ROC curves for the classification performance of the 29 subjects. (a) ROC curves for all filtering approaches: NA-MEMD, EEMD, EMD, the IIR filter and the CWT. The AUROC using NAMEMD is larger than the other AUROCs. (b) Standard errors of the AUROCs of the 29 subjects, which illustrate higher AUROCs of NA-MEMD than the other methods

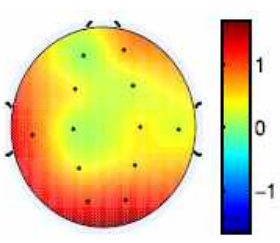

(a) EEG Sources for left-hand MI

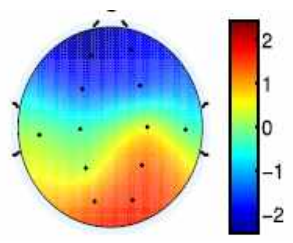

(b) EEG Sources for right-hand MI
Fig. 11. EEG source headmaps estimated using averaged common spatial patterns across the 29 subjects. The dots represent the locations of the 12 channel electrodes used for the analysis. Note the ipsilateral EEG sources corresponding to the MI tasks
Fig. 11 and 12 shows the EEG amplitude source images calculated using the source separation algorithm, common spatial patterns [1], which has often been applied to the BCI systems. Fig. 11 displays the average EEG source images across 29 subjects, and the well-known ipsilateral increase of the sources corresponding to the MI tasks can be noted [26]. In particular, the source image of the subject 23 clearly illustrates the dominant sources on the central region in Fig. 12. Fig. 13 illustrates the causality networks of the brain responses during the MI tasks using the average NA-MEMD PDC features for the 29 subjects. In particular, the network diagram only displays the $10 \%$ highest PDC features among all 66 PDC directional features from the pairwise combinations of 12 EEG channels. The study of the event-related synchronization/ desynchronization demonstrated the ipsilateral and contralateral responses of EEG to the MI tasks [35]. Additionally, Chung et al. [36] demonstrated the contralateral connectivity during MI of the left and right hands. Fig. 13 also shows the lateral differences in PDC features corresponding to the MI tasks, namely, $\pi_{\mathrm{C} 3, \mathrm{P} 4}^{\max }$ during left-hand $\mathrm{MI}$ and $\pi_{\mathrm{C} 4, \mathrm{P} 3}^{m a x}$ during right-hand $\mathrm{MI}$.

The performance of NA-MEMD depending on the SNR and channel number of noise in the additional channels was

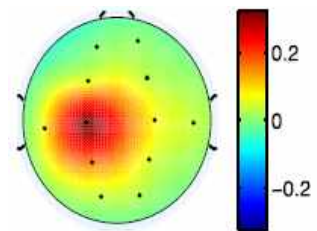

(a) EEG Sources for left-hand MI

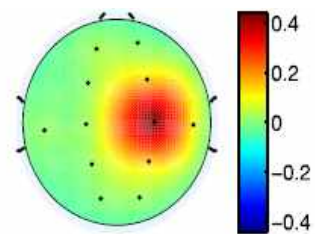

(b) EEG Sources for right-hand MI
Fig. 12. EEG source headmaps estimated using the common spatial patterns of the subject 23. The dots represent the locations of the 12 channel electrodes used for the analysis. Note the ipsilateral EEG sources corresponding to the MI tasks

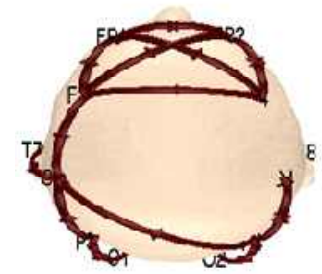

(a) EEG Sources for left-hand MI

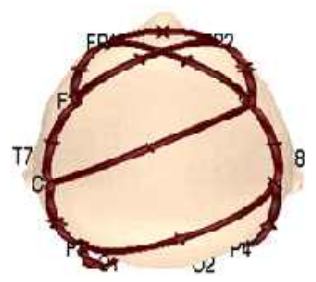

(b) EEG Sources for right-hand $\mathrm{MI}$
Fig. 13. PDC headmaps estimated using averaged NAMEMD PDC features across the 29 subjects, which show the highest $10 \%$ PDC features among all 66 PDC directional features from the pairwise combinations of 12 EEG channels. Lateral differences can be found in the PDC features for the left-hand and right-hand MI tasks 


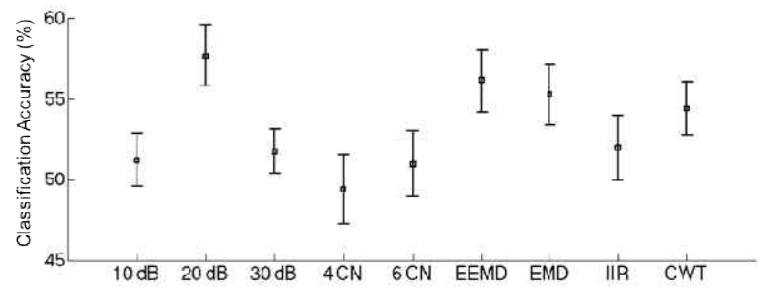

Fig. 14. Studies of NA-MEMD performance depending on the amount of noise in the additional channels and the number of noise channels for the MI dataset. ' $10 \mathrm{~dB}$ ', ' $20 \mathrm{~dB}$ ' and ' $30 \mathrm{~dB}$ ' denote the SNR of noise in the additional channels of NA-MEMD, and ' $4 \mathrm{CN}$ (channel noise)' and ' $6 \mathrm{CN}$ ' the number of noise channels with $20 \mathrm{~dB}$ SNR. Note that NAMEMD with ' $20 \mathrm{~dB}$ ' SNR and two additional noise channel produced the best performance

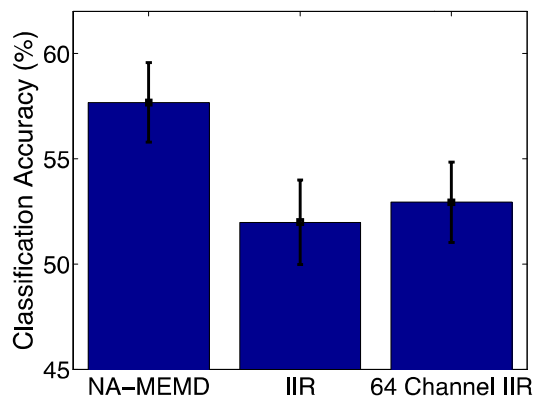

Fig. 15. The results of all 64 channel EEG data. Although the results of the 64 channel EEG using the IIR filter outperformed those of the 12 channel EEG, they were still significantly worse than those of 12 channel EEG using NA-MEMD $(\mathrm{p}<0.05)$

investigated like the simulation in Fig. 4. Fig. 14 illustrates the classification rates produced using NA-MEMD with different conditions of the additional channel noise like ' 10 $\mathrm{dB}$ ', '30 dB', '4 $\mathrm{CN}$ (channel noise)' and ' $6 \mathrm{CN}$ ', while the other classification rates are the same as those in Fig. 6. This experiment demonstrates NA-MEMD with ' $20 \mathrm{~dB}$ ' SNR and two noise channels derived the best performance for the MI EEG dataset.

Lastly, 64 channel EEG data was tested using the IIR filter and the performance of the 29 subjects is shown in Fig. 15. Although the performance with more number of channel data was slightly higher than that of the 12 channel data using the IIR filter, NA-MEMD with 12 channel data still outperformed the conventional IIF filter significantly $(p<0.05)$, proving the superiority of the proposed approach compared to the conventional method.

\section{Discussion}

We presented the directionality of neural oscillations during MI tasks using partial directed coherence with various frequency decomposition algorithms. Classification analysis using a SVM demonstrated that the data-driven EMD algorithms outperform the conventional bandpass filtering algorithm. Furthermore, NA-MEMD was shown to be the optimal method for estimating an accurate PDC feature due to its consideration of multichannel information. For the future work, different methods of investigating the brain connectivity, such as the original Granger causality [7] and directed transfer function (DTF) [37], will also be applied to obtain brain connectivity information during MI tasks and to observe various aspects of the causal network. These further investigations of the various pieces of directional connectivity information would uncover more detailed information regarding the functional connectivity of brain regions during MI and planning tasks.

This study focused on an accurate frequency filtering algorithm to estimate PDC features and thus did not consider any source separation method to extract the main sources of multichannel EEG data. Advanced blind source separation methods, such as independent component analysis and complex common spatial patterns algorithms [38][39], will be considered to improve the separation of the MI tasks. In addition, the PDC headmap study in Fig. 13 will be investigated in further detail for each individual subject to verify the brain causal network during MI and planning tasks in future work. NA-MEMD would provide more accurate brain connectivity information than any other conventional method, linear and single-channel frequency filtering algorithms.

\section{Conclusion}

We have presented an accurate PDC feature extraction method using NA-MEMD for synthetic data and a realworld EEG data of 29 subjects during MI tasks. In particular, PDC measurement with time delays and a machine learning algorithm, support vector machine, clearly demonstrated the improved classification accuracy using NA-MEMD compared with the other EMD algorithms and the conventional bandpass filter techniques. Therefore, a multivariate algorithm considering multichannel data is an essential prerequisite for estimating the network/ connectivity among multichannel data.

\section{Acknowledgements}

The present research has been supported by the Research Grant of Kwangwoon University in 2015, Basic Science Research Program through the National Research Foundation of Korea(NRF) funded by the Ministry of Education, Science and Technology (2014R1A1A2059483) and Institute for Information \& communications Technology Promotion (IITP) grant funded by the Korea government (MSIP) (No.B0184-15-1003, The Development of oneM2M Conformance Testing Tool and QoS Technology). 


\section{References}

[1] C. Park, C. C. Took, and D. P. Mandic, "Augmented complex common spatial patterns for classification of noncircular EEG from motor imagery tasks," IEEE Trans. on Neural Systems and Rehabilitation Engineering, vol. 22, no. 1, pp. 1-10, Dec. 2013

[2] C. Park, D. Looney, P. Kidmose, M.Ungstrup and D.P. Mandic "Time-Frequency Analysis of EEG Asymmetry using Bivariate Empirical Mode Decomposition" IEEE Transactions on Neural Systems and Rehabilitation Engineering, vol. 19, no. 4, pp. 366-373, 2011

[3] K. Sivasankari and K. Thanushkodi, "An Improved EEG Signal Classification Using Neural Network with the Consequence of ICA and STFT," Journal of Electrical Engineering and Technology, vol. 9, no. 3, pp. 1060-1071, 2014

[4] X. Li, S. O, Y. Pan and K. Ang, "Connectivity pattern modeling of motor imagery EEG," in Proc. Computational Intelligence, Cognitive Algorithms, Mind, and Brain (CCMB), 2013, pp, 16-19

[5] C. Park, D. Looney, N. Rehman, A. Ahrabian and D. P. Mandic, "Classification of motor imagery BCI using multivariate empirical mode decomposition," IEEE Trans. on Neural Systems and Rehabilitation Engineering, vol. 21, no. 1, pp. 10-22, Jan. 2013

[6] A. Solodkin, P. Hlustik, E. E. Chen and S. L. Small, "Fine modulation in network activation during motor execution and motor imagery," Cerebral Cortex, vol. 14, pp. 1246-55, Nov. 2004

[7] C. W. J. Granger, "Investigating causal relations by econometric models and cross-spectral methods," Econometrica," vol. 37, no. 3, pp. 424-438, Aug. 1969

[8] A. N. Silchenko, I. Adamchica, N. Pawelczyka, C. Hauptmanna, M. Maaroufb, V. Sturmb and P. A. Tassa, "Data-driven approach to the estimation of connectivity and time delays in the coupling of interacting neuronal subsystems," Journal of Neuroscience Methods, vol. 191, no. 1, pp. 32-44, Aug. 2010

[9] K. Sameshima and L. A. Baccal, "Using partial directed coherence to describe neuronal ensemble interactions," Journal of Neuroscience Methods, vol. 94, no. 1, pp. 93-103, Dec. 1999

[10] N. E. Huang, Z. Shen, S. R. Long, M. L. Wu, H. H. Shih, Z. Quanan, N. C. Yen, C. C. Tung and H. H. Liu, "The empirical mode decomposition and the Hilbert spectrum for nonlinear and non-stationary time series analysis," Proceedings of the Royal Society A vol. 454, pp. 903-95, Mar. 1998

[11] C. Park, M. Plank, J. Snider, S. Kim, H. Huang, S. Gepshtein, T. Coleman, H. Poizner, "EEG gamma band oscillations differentiate the planning of spatially directed movements of the arm versus eye: multivariate empirical mode decomposition analysis," IEEE Transactions on Neural Systems and Rehabilitation Engineering, vol. 22, pp. 1083-96, 2014

[12] S. Lahmiri and M. Boukadoum, "A weighted biosignal denoising approach using empirical mode decomposition," Biomedical Engineering Letters, vol. 5, no. 2, pp. 131-139, 2015

[13] D. Looney and D. P. Mandic, "Multi-scale image fusion using complex extensions of EMD," IEEE Transactions on Signal Processing," vol. 57 pp. 162630, 2009

[14] N Rehman and D. P. Mandic, "Multivariate empirical mode decomposition," Proceedings of the Royal Society A, vol. 466, pp. 1291-302, 2010

[15] J. Rodrigues and A. Andrade, "Instantaneous Granger Causality with the Hilbert-Huang Transform," ISRN Signal Processing, pp. 1-9, 2013

[16] Z. Wu and N. E. Huang, "Ensemble empirical mode decomposition:A noise-assisted data analysis method," Advances in Adaptive Data Analysis, vol. 1, no. 1, 2009

[17] J. Cui J and W. Freeden, "Equidistribution on the sphere," Journal on Scientific Computing, vol. 18, pp. 595-609, 1997

[18] N. Rehman and D. P. Mandic, "Filter bank property of multivariate empirical mode decomposition," IEEE Transactions on Signal Processing, vol. 59, 2421-6, 2011

[19] G. Rilling, P. Flandrin and P. Goncalves, "On empirical mode decomposition and its algorithms," Proceedings of the IEEE-EURASIP Workshop on Nonlinear Signal and Image Processing (NSIP), vol. 3, pp. 8-11, 2003

[20] N. E. Huang, M. L. Wu, S. R. Long, S. S. P. Shen, W. Qu W, P. Gloersen and K. L. Fan, "A confidence limit for the empirical mode decomposition and Hilbert spectral analysis," Proceedings of the Royal Society A, vol. 459,pp. 2317-45, 2003

[21] L. A. Baccal, and K. Sameshima, "Partial directed coherence: a new concept in neural structure determination," Biological Cybernetics, vol. 84 pp. 463-74, 2001

[22] G. Schalk, D. McFarland, T. Hinterberger, N. Birbaumer and J. R. Wolpaw, "BCI2000: A general purpose brain-computer interface (BCI) system," IEEE Transactions on Biomedical Engineering, vol. 51, pp. 1034-43, 2004

[23] A. Goldberger, L. Amaral, L. Glass, J. Hausdorff, P. Ivanov, R. Mark, J. Mietus, G. Moody, C. K. Peng and H. Stanley, "PhysioBank, PhysioToolkit, and PhysioNet: Components of a new research resource for complex physiologic signals Circulation," 101 e215-e220

[24] M. Arvaneh, C. Guan, K. K. Ang and C. Quek, "Optimizing the channel selection and classification accuracy in EEG-Based BCI," IEEE Transactions on 
Biomedical Engineering, vol. 58, no. 6, pp. 18651873, 2011

[25] H. Ramoser, J. Muller-Gerking and G. Pfurtscheller, "Optimal spatial filtering of single trial EEG during imagined hand movement," IEEE Transactions on Rehabilitation Engineering, vol. 8, pp. 441-6, 2000

[26] J. Muller-Gerking, G. Pfurtscheller and H. Flyvbjerg, "Designing optimal spatial filters for single-trial EEG classification in a movement task," Clinical Neurophysiology, vol. 110, pp. 787-98, 1999

[27] K. Lugger, D. Flotzinger, A. Schlögl, M. Pregenzer and G. Pfurtscheller, "Feature extraction for on-line EEG classification using principal components and linear discriminants," Medical \& Biological Engineering \& Computing, vol. 36, pp. 309-314, 1998

[28] R. Noori, A. R. Karbassi, A. Moghaddamnia, D. Han, M. H. Zokaei-Ashtiani, A. Farokhnia and M. G. Gousheh, "Assessment of input variables determination on the SVM model performance using PCA, Gamma test, and forward selection techniques for monthly stream flow prediction," Journal of Hydrology, vol. 401, no. 3-4, pp. 177-189, 2011

[29] R. E. Bellman, "Dynamic Programming" North Chelmsford, MA: Courier Dover Publications

[30] H. Drucker, C. J. Burges, L. Kaufman, A. Smola and V. Vapnik, "Support Vector Regression Machines," Cambridge, MA: MIT Press

[31] S. Canu, Y. Grandvalet, V. Guigue and A. Rakotomamonjy, "SVM and kernel methods Matlab toolbox", Perception Systems et Information (Rouen, France: INSA de Rouen, 2005

[32] G. R. Müller-Putz, R. Scherer, C. Brunner, R. Leeb, and G. Pfurtscheller, "Better than random? a closer look on BCI results," International Journal of Bioelectromagnetism, vol. 10, no. 1, pp. 52-55, 2008

[33] M. Ahn and S. Jun, "Performance variation in motor imagery brain-computer interface: A brief review," Journal of Neuroscience Methods, vol. 243, pp. 103110,2015

[34] J. A. Hanley and B. J. McNeil,, "A method of comparing the areas under receiver operating characteristic curves derived from the same cases," Radiology, vol. 148, pp. 839-43, 1983

[35] H. Yuan, A. Doud, A. Gururajan and B. He, "Cortical imaging of event- related (de)synchronization during online control of brain-computer interface using minimum-norm estimates in frequency domain," IEEE Transactions on Neural Systems and Rehabilitation Engineering, vol. 16, pp. 425-31, 2008

[36] Y. Chung, J. Kang and S. Kim, "Analysis of correlated EEG activity during motor imagery for brain computer interfaces," in Proceedings of Control, Automation and Systems (ICCAS), pp. 337-41, 2011

[37] M. Kaminski, M. Ding, W. A. Truccolo and S. Bressle, "Evaluating causal relations in neural systems : Granger causality, directed transfer function and statistical assessment of significance," Biological Cybernetics, vol. 85, pp. 145-57, 2011

[38] Y. Kim and C. Park, "Strong Uncorrelated Transform Applied to Spatially Distant Channel EEG Data," IEIE Transactions on Smart Processing and Computing, vol. 4 , pp. 97-102, Apr. 2015

[39] S. Ge, M. Han and X. Hong, "A fully automatic ocular artifact removal from EEG based on fourthorder tensor method," Biomedical Engineering Letters, vol. 4, no. 1, pp. 55-63, Mar. 2014

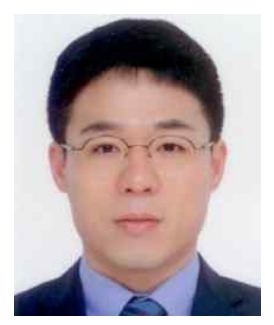

Ki-Baek Lee He received the B.S. and Ph.D. degrees in Electrical Engineering from Korea Advanced Institute of Science and Technology (KAIST), Daejeon, Korea, in 2005 and 2014, respectively. Since 2014, he has been an assistant Professor with the Department of Electrical Engineering, Kwangwoon University, Seoul, Korea. He has researched computational intelligence, in particular, in the area of swarm intelligence and multi-objective evolutionary algorithms (MOEAs). His research interests also include a real world application such as on-line multi-objective evolutionary navigation for humanoid robots.

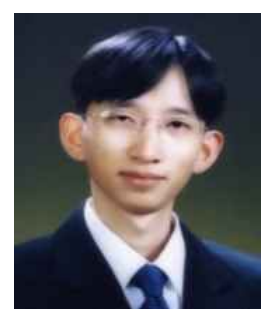

Ko Keun Kim He received the Ph.D degree in Biomedical Engineering from Seoul National University, Seoul, Republic of Korea, in 2010. He is currently a Chief Research Engineer in Intelligence Lab., Convergence Center at LG Electronics, Seoul, Republic of Korea. Prior to his current position, he was a Research Fellow of Center for Cognition and Sociality at Institute for Basic Science, Daejeon, Republic of Korea.

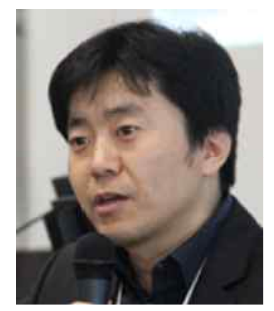

Jaeseung Song $\mathrm{He}$ is an assistant professor in the Computer and Information Security Department at Sejong University. He holds the position of oneM2M Test Working Group chair. Prior to his current position, he worked for NEC Europe Ltd. and LG Electronics in various positions. $\mathrm{He}$ received a $\mathrm{Ph}$.D. from Imperial College London in the Department of Computing, United Kingdom. He holds B.S. and M.S. degrees in computer science from Sogang University. 


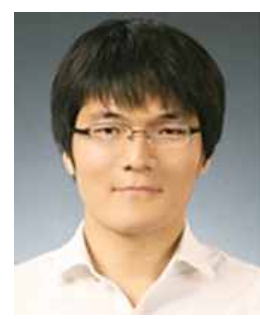

Jiwoo Ryu He received BS and MS degrees in computer engineering from Kwangwoon university in Seoul, South Korea, in 2011 and 2015, respectively. His research interests include biomedical signal processing and image processing.

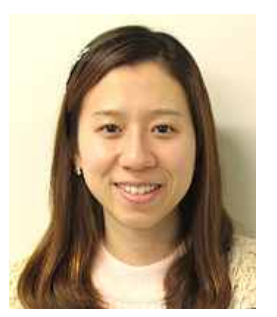

Youngjoo Kim She received her B.S. degree in Computer Engineering from Kwangwoon University, Seoul, South Korea, in 2015. Currently, she is a graduate student in the Bio-Medical Computing Laboratory (BMCL), Department of Computer Engineering, Kwangwoon University, Seoul, South Korea. Her research interests include bio-medical signal processing, machine learning and statistical analysis.

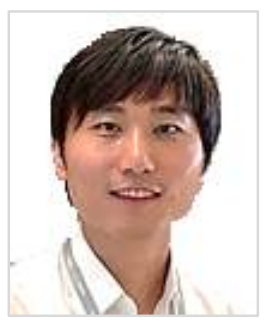

Cheolsoo Park He is an assistant professor of Computer Engineering Dept. at Kwangwoon University, Seoul, South Korea. He received the B. Eng. degree in electrical engineering from Sogang University, Seoul, and the M. Sc. degree in biomedical engineering department from Seoul National University, South Korea. In 2012, he received his $\mathrm{PhD}$ degree in adaptive nonlinear signal processing from Imperial College London, London, U.K and worked as a postdoctoral researcher in bioengineering department at University California, San Diego, U.S. His research interests are mainly in the area of machine learning, adaptive and statistical signal processing, with applications in brain computer interface, computational neuroscience and wearable technology. 